\title{
Capacitação Profissional na Geração de Resíduos Sólidos de Serviço de Saúde em Ambiente Hospitalar
}

\section{Professional Training in the Solid Residues Generation from Health Services in a Hospital Environment}

\author{
Huama Monteiro de Brito ; Marcelo Dias de Souza*b; Patricia Paz da Costac; Géssica Lataliza de Menezes; \\ Flávia Machado Coelho ; Bruna Karoline de Almeida Santiago ${ }^{a}$
}

\author{
anniversidade de Cuiabá, Programa de Pós-Graduação Stricto Sensu em Ambiente e Saúde. MT, Brasil. \\ bUniversidade de Cuiabá, Programa de Pós-Graduação Stricto Sensu em Ciências Ambientais. MT, Brasil. \\ 'Universidade de Cuiabá. MT, Brasil. \\ *E-mail: marcelo.dsouza@kroton.com.br
}

\begin{abstract}
Resumo
O trabalho teve como objetivo analisar a geração de resíduos comuns do Hospital São Luiz de Cáceres do Estado de Mato Grosso, em relação à capacitação dos funcionários no local de trabalho. Trata-se de estudo de intervenção, analítico, experimental de resíduos de serviços de saúde. A realização da capacitação foi no ano de 2017, em que foram abordados cinco pontos de geração no hospital, tendo como prioridade a quantidade de resíduos gerados em cada ponto, sendo quantificados e analisados antes e após a capacitação profissional dos colaboradores. Os resultados demonstraram que os resíduos comuns gerados no local estão sendo descartados de forma incorreta, pois foram observados resíduos da classe A nas lixeiras de classe $\mathrm{D}$, sendo que mesmo com a capacitação, a segregação ainda continuou sendo realizada de forma incorreta por alguns profissionais. Portanto, em função de danos financeiros e ambientais, pode-se dizer que é de suma importância que os profissionais sejam capacitados por uma pessoa qualificada. É necessária uma pessoa capacitada para se responsabilizar pelo PGRSS, SCIH e gestores, aplicando treinamento de capacitação e monitorando diretamente o manejo dos resíduos sólidos gerados no ambiente hospitalar.
\end{abstract}

Palavras-chave: Lixo Hospitalar. Segregação. Gerenciamento de Resíduos.

\begin{abstract}
The work aimed to analyze the common residues generation of Hospital São Luiz de Cáceres of the state of Mato Grosso, in relation to the employees training in the workplace. This is an intervention, analytical and experimental study of health services residues. The training was carried out in 2017, in which five generation points were added, having as priority the amount of residues generated in each point, being quantified and advanced and after the professional employees training. The results showed that the common residues generated at the site is being disposed of incorrectly, because class A residues were observed in class D dumps, being that even with the training, the segregation still continued to be performed incorrectly by some professionals. Therefore, due to the financial and environmental damage, it can be said that it is extremely important that professionals are trained by a qualified person. A trained person is required to be responsible for the PGRSS, SCIH and managers, applying training and monitoring directly the solid residues management in the hospital environment.
\end{abstract}

Keywords: Hospital Garbage. Segregation. Residues Management.

\section{Introdução}

Os RSS, conhecidos como Resíduos de Serviços de Saúde são gerados por prestadores de assistência à saúde, como por exemplo, odontológica, laboratorial, farmacêutica e instituições de ensino e pesquisa médica relacionada tanto à população humana quanta à veterinária. Os RSS, apesar de serem representados por uma pequena parcela em relação à quantidade de resíduos gerados em uma comunidade, são fontes potenciais de propagação de doenças e apresentam um risco aos trabalhadores dos serviços de saúde e à comunidade em geral, quando gerenciados de forma inadequada (BRASIL, 1993).

O conhecimento sobre os tipos de resíduos gerados no estabelecimento é de suma importância para a criação de programas, que possam avaliar as condições de alguns resíduos e a forma de tratamento para cada tipo, conforme classificação estabelecida na RDC ANVISA $n^{\circ}$ 306. Esta resolução estabelece definições, classificação e procedimentos mínimos para o gerenciamento dos RSS, sendo os resíduos classificados segundo seus riscos (BRASIL, 2004).

É inquestionável a necessidade de implantar políticas de gerenciamento dos resíduos sólidos de serviços de saúde (RSSS) nos vários estabelecimentos de saúde, não apenas investindo na organização e sistematização dessas fontes geradoras, mas, fundamentalmente, mediante o despertar de uma consciência humana e coletiva quanto à responsabilidade com a própria vida humana e com o ambiente. Nesse sentido, os profissionais devem ter melhor conhecimento sobre os resíduos gerados por suas atividades, objetivando minimizar riscos ao ambiente e à saúde dos trabalhadores, bem como da população em geral. Isso depende, em parte, da formação desses profissionais.

Entre as fontes de degradação ambiental, os resíduos gerados na área da saúde representam uma peculiaridade importante, quando gerenciados inadequadamente, pois 
alguns resíduos perigosos podem oferecer risco ao ambiente e para a saúde humana. Essa problemática vem sendo cada vez mais objeto de preocupação de órgãos de saúde, ambientais, prefeituras, técnicos e pesquisadores da área. Isso se verifica pela quantidade de legislações e referências existentes, que preconizam condutas de gerenciamento dos resíduos nos locais nos quais são prestados serviços à saúde (COELHO, 2000).

Em ambiente hospitalar, os RSS se constituem em um problema sério, em função, principalmente, de falta de informações, gerando mitos entre funcionários, pacientes e a comunidade. Assim, demonstra-se o quanto é necessário o desenvolvimento de diferentes práticas de gestão de resíduos como o Plano de Gerenciamento de Resíduos de Serviços de Saúde (PGRSS), que pode contribuir com a redução da quantidade de resíduos segregados de forma inadequada (NAIME, 2008).

O PGRSS é um documento integrante do processo de licenciamento ambiental, sendo definido como um conjunto de procedimentos de gestão, que visam o correto gerenciamento dos resíduos produzidos no estabelecimento. São planejados e executados pelo responsável legal do estabelecimento gerador, baseados em diretrizes científicas, normativas e legais (BRASIL, 2010). O plano visa minimizar a produção de resíduos, proporcionando um encaminhamento seguro e com eficiência, minimizando impactos ambientais e humanos no local e vizinhança, assim garantindo a integridade física, direta e indiretamente, dos envolvidos (BRASIL, 2006).

As soluções para minimizar a problemática da má gestão dos resíduos sólidos de serviços de saúde dependem de uma série de decisões tomadas em diferentes níveis do sistema dos profissionais, que trabalham no local (SCHNEIDER et al., 2002; COELHO, 2003). Portanto, o estudo teve como objetivo analisar a geração de resíduos classe D (comum) do Hospital São Luiz de Cáceres do Estado de Mato Grosso, em relação à capacitação profissional sobre o manejo dos RSS aplicados aos funcionários no local de trabalho.

\section{Desenvolvimento}

\subsection{Descrição e área de estudo}

Trata-se de um estudo de intervenção analítica experimental com base em ensaio para análise do PGRSS do Hospital São Luiz de Cáceres. O local de estudo se localiza na cidade do município de Cáceres - Mato Grosso, localizada na região Centro-sul do Estado e na microrregião do Alto Pantanal. Tendo uma população aproximada de 90.881 habitantes, segundo ou IBGE, em 2016, e desta forma fazendo fronteira com a Bolívia. A pesquisa foi elaborada no Hospital São Luiz (HSL), que presta serviço às diversas especialidades de saúde do município de Cáceres e outras regiões de MT.

\subsection{Procedimentos de coleta e amostragem}

O HSL conta com 154 leitos, sendo que os pontos de geração analisados foram amostrados por dois meses - novembro e dezembro de 2017 e maio e junho de 2018, após a capacitação. Para ambas as etapas, nas duas primeiras semanas do mês, foram analisadas em quatro dias da semana, sendo que em cada dia sorteado três pontos de geração para quantificação e análise, em que ao final da amostragem, cada um dos pontos foi analisado duas vezes, totalizando assim, quatro amostras para cada ponto de geração, sendo realizado por dois meses, assim foram obtidas quatro amostras para cada mês, portanto, oito amostras para cada etapa (antes e após a capacitação).

A pesquisa foi dividida em três etapas: quantificação e análise do gerenciamento de resíduos sólidos de serviços de saúde da classe D (comum); capacitação dos profissionais geradores de resíduos de serviços de saúde; reanálise do gerenciamento de resíduos sólidos de serviços de saúde póscapacitação. Sendo analisados os pontos de geração:

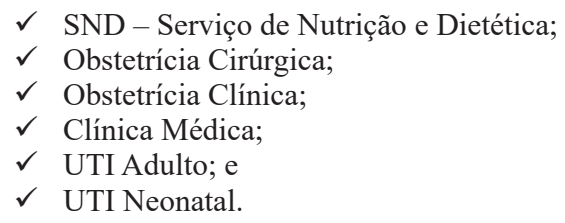

O estudo foi submetido à apreciação do Comitê de Ética em Pesquisa da Universidade de Cuiabá - CEP/UNIC, conforme os critérios previstos em normatização, cumprindo assim todos os requisitos necessários à realização de pesquisas com seres humanos determinados na Resolução 466/12 do Conselho Nacional de Saúde (CNS). A coleta de dados, bem como a capacitação só foram realizadas após aprovação do estudo pelo Comitê de Ética e Pesquisa em Seres Humanos da UNIC.

Os profissionais, que participaram da pesquisa, foram identificados por meio da escala de trabalho dos colaboradores pertencentes ao HSL, sendo utilizado o método de amostragem aleatória estratificada, tendo por estratos os pontos de geração, nos quais foram passados aos profissionais os objetivos e benefícios da participação na pesquisa, através do termo de consentimento livre e esclarecido, que assinaram concordando com o trabalho.

Para a capacitação profissional, as equipes de cada setor receberam orientações sobre o PGRSS, envolvendo todo o processo de compreensão e reflexão crítica das normas de biossegurança e preservação ambiental dos RSS. Para os profissionais do plantão ímpar, foi proposto que um funcionário por equipe, de cada setor, participasse da brincadeira: "Amigo verde", durante 20 dias, em que este realizou a fiscalização de modo voluntário dos descartes de resíduos da equipe com quem trabalha durante os plantões, durante as fiscalizações eles realizavam orientações, e enviavam fotos dos descartes via celular, para que todos os "amigos verdes" trocassem experiências.

Após a capacitação dos profissionais de cada setor envolvido na pesquisa, o processo estabelecido na primeira 
etapa foi realizado, novamente, com intuito de analisar as melhorias obtidas com a capacitação.

\subsection{Análises de dados}

Os dados foram tabulados e analisados graficamente, bem como foi feita análise de estatística descritiva para os dados coletados da quantificação dos resíduos. Para analisar o efeito da capacitação, as médias da quantidade gerada dos resíduos foram comparadas pelo teste $\mathrm{T}$ de Student, considerando as duas amostras presumindo variâncias equivalentes, assim considerando uma probabilidade de erro de $5 \%$.

\subsection{Resultados e Discussão}

Verificou-se que a separação dos resíduos ocorre no momento de sua geração, visto que os setores possuem lixeiras para resíduos comuns. No entanto, mesmo com as lixeiras destinadas para cada tipo de resíduo, o descarte não era realizado de forma correta. Contudo, nos meses estudados, o total de resíduos comuns do grupo D coletado foi de $461,3 \mathrm{~kg}$, a média de geração entre os meses novembro e dezembro de 2017 dos setores se mantiveram. Porém, o setor de UTI Neonatal foi o que menos gerou resíduos desse grupo (Figura 1).
Figura 1 - Gráfico da composição total dos resíduos de serviços de saúde pertencentes ao grupo D em KG, gerados nos setores do Hospital São Luiz - Cáceres/MT - novembro e dezembro/2017.

\section{Composição total dos RSS - Grupo D - Antes da}

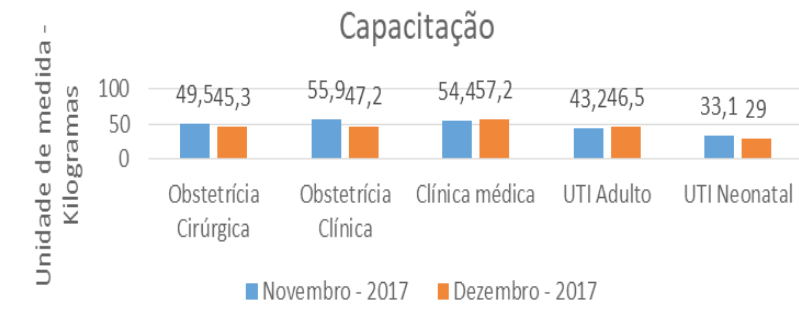

Fonte: Dados da pesquisa.

O setor que gerou maior quantidade de resíduos comuns durante a coleta, antes da capacitação, foi o setor de clínica médica, todavia, os valores não representam o valor total gerado pelo setor, pois a segregação não foi realizada de forma adequada. Além disso, notou-se que não foram descartados nas lixeiras apenas os resíduos do grupo $\mathrm{D}$, mas de vários grupos juntos.

Com a avaliação desta etapa, foi realizada a segregação dos resíduos, em que dos 461,3 kg pesados, apenas 362,26 kg realmente pertencem ao grupo de resíduos comuns, os demais se dividem entre 59,98 $\mathrm{kg}$ de resíduos infectantes e 39,100 kg de resíduos orgânicos (Quadro 1).

Quadro 1 - Composição média dos resíduos de serviços de saúde pertencentes ao grupo D em KG, após segregação correta nos setores do Hospital São Luiz antes da capacitação. Cáceres-MT, 2018.

\begin{tabular}{|c|c|c|c|c|c|c|c|c|c|}
\hline \multirow{2}{*}{$\begin{array}{l}\text { Unidade } \\
\text { Geradora }\end{array}$} & \multicolumn{3}{|c|}{ Infectante } & \multicolumn{3}{|c|}{ Comum } & \multicolumn{3}{|c|}{ Orgânico } \\
\hline & $\sum$ & $\bar{X}$ & CV $(\%)$ & $\sum$ & $\bar{X}$ & CV $(\%)$ & $\sum$ & $\bar{X}$ & CV $(\%)$ \\
\hline Obstetrícia cirúrgica & 9,3 & 1,16 & 30,47 & 77,6 & 9,7 & 20,01 & 7,9 & 0,98 & 59,26 \\
\hline Obstetrícia clínica & 10,5 & 1,31 & 47,08 & 83,65 & 10,45 & 21,78 & 9,2 & 1,15 & 69,55 \\
\hline Clínica médica & 18,1 & 2,26 & 24,88 & 85,6 & 10,7 & 16,30 & 7,9 & 0,98 & 38,34 \\
\hline UTI Adulto & 12,78 & 1,59 & 23,30 & 66,66 & 8,33 & 6,29 & 10,3 & 1,28 & 32,86 \\
\hline UTI neonatal & 9,3 & 1,16 & 30,39 & 48,75 & 6,09 & 13,95 & 4,05 & 0,50 & 63,44 \\
\hline
\end{tabular}

Fonte: Dados da pesquisa.

Após a segregação correta se observou que os setores: Obstetrícia cirúrgica, Obstetrícia clínica e UTI neonatal apresentaram coeficiente de variação de alta dispersão, no que diz respeito aos resíduos orgânicos descartados. Embora sejam classificados como resíduos do grupo D, os setores abertos possuem coletores específicos para estes resíduos, o que não justifica o coeficiente de variação de alta dispersão.

Quanto aos resíduos infectantes, esses demonstram sempre estar presentes nas lixeiras classe D, o que também não se justifica, uma vez que os setores têm sacos coletores para esses resíduos. A separação de cada resíduo deve estar de acordo com sua classificação, com a reciclagem e com a possibilidade de reciclagem entre esses grupos, classificada como comuns e os recicláveis (SOUZA, 2006).

A segregação criteriosa que, muitas vezes, é realizada em apenas uma pequena parte dos RSSS é necessitário submeter a todos os resíduos comuns, destinados ao aterro sanitário, reduzindo os custos operacionais e os riscos à saúde pública
(DIAS; FIGUEIREDO, 1999). De acordo com Silva e Hoppe (2006), as instituições de saúde têm se preocupado com a segregação e depósito de resíduos pertencentes ao grupo A, porém os demais resíduos ganham pouca atenção.

Cabe ressaltar que resíduos do grupo A, descartados nos recipientes do grupo $\mathrm{D}$, podem causar impactos para a saúde e o meio ambiente, trazendo impactos ambientais como de vizinhança, podendo infectar pessoas como catadores de resíduos ou os profissionais responsáveis pela coleta dos resíduos domiciliares, encaminhados para os aterros municipais.

Depois da realização completa da etapa de quantificação, mas agora após a capacitação profissional, o valor total de resíduos comuns gerados foi de $443,6 \mathrm{~kg}$. Os setores avaliados durante a pesquisa seguem mantendo a média de geração de resíduos pertencentes ao grupo D. Quando comparados entre si, o setor de UTI Neonatal continua sendo o que menos gera resíduos do grupo D (Figura 2). 
Figura 2 - Gráfico da composição total dos resíduos de serviços de saúde pertencentes ao grupo D em KG, gerados nos setores do Hospital São Luiz - Cáceres/MT - maio e junho/2018.

\section{Composição total dos RSS - Grupo D - Após a \\ Capacitação}

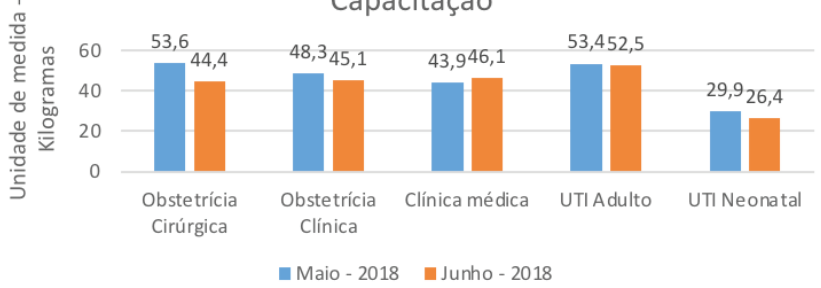

Fonte: Dados da pesquisa.
De acordo com 443,6 $\mathrm{kg}$ pesados, apenas $378,5 \mathrm{~kg}$ realmente pertencem ao grupo de resíduos comuns, os demais se dividem entre $34,2 \mathrm{~kg}$ de resíduos infectantes e 30,9 kg de resíduos orgânicos. Antes da capacitação, o valor total dos resíduos do grupo D foi de $461,3 \mathrm{Kg}$, deste, $13 \%$ estava composto por resíduos do grupo A e 8,47\% composto por resíduos orgânicos. Após a capacitação, o valor total dos resíduos do grupo D foi de $443,6 \mathrm{~kg}$, sendo que $7,7 \%$ composto por resíduos do grupo A e 6,96\% composto por resíduos orgânicos (Quadro 2).

Quadro 2 - Composição média dos resíduos de serviços de saúde pertencentes ao grupo D em KG, após segregação correta nos setores do Hospital São Luiz após a capacitação

\begin{tabular}{|c|c|c|c|c|c|c|c|c|c|}
\hline \multirow{2}{*}{$\begin{array}{l}\text { Unidade } \\
\text { Geradora }\end{array}$} & \multicolumn{3}{|c|}{ Infectante } & \multicolumn{3}{|c|}{ Comum } & \multicolumn{3}{|c|}{ Orgânico } \\
\hline & $\sum$ & $\bar{X}$ & $\mathrm{CV}(\%)$ & $\sum$ & $\bar{X}$ & $\mathrm{CV}(\%)$ & $\sum$ & $\bar{X}$ & $\mathrm{CV}(\%)$ \\
\hline Obstetrícia cirúrgica & 4,5 & 0,59 & 21,64 & 91,1 & 11,38 & 18,87 & 2,4 & 0,3 & 65,65 \\
\hline Obstetrícia clínica & 9,3 & 1,16 & 31,45 & 72,8 & 9,1 & 12,82 & 11,3 & 1,41 & 31,32 \\
\hline Clínica médica & 10,4 & 1,3 & 39,43 & 75,3 & 9,41 & 10,61 & 4,3 & 0,53 & 58,78 \\
\hline UTI Adulto & 4,9 & 0,61 & 34,65 & 92 & 11,5 & 16,24 & 9 & 1,12 & 45,44 \\
\hline UTI neonatal & 5,1 & 0,63 & 34,76 & 47,3 & 5,91 & 9,62 & 3,9 & 0,48 & 52,79 \\
\hline
\end{tabular}

Fonte: Dados da pesquisa.

O coeficiente de variação se manteve em baixa dispersão apenas no setor de Obstetrícia cirúrgica no que se refere à presença de resíduos infectantes descartados nos coletores para resíduos comuns. Em relação ao descarte dos resíduos orgânicos, em meio ao grupo $\mathrm{D}$, não houve mudanças em relação aos valores obtidos antes da capacitação, todos os setores apresentaram coeficiente de variação de alta dispersão. Mesmo com diminuição dos resíduos do grupo $\mathrm{A}$ e dos resíduos orgânicos, em meio aos resíduos comuns, pode-se perceber que ainda existem estes resíduos segregados junto com os resíduos comuns.

Entre os resíduos do grupo A, comumente encontrados, se pode destacar: presença de luvas de procedimento; materiais de curativos contendo sangue; e as lancetas/agulhas. Este fato demonstra a importância do monitoramento da gestão de resíduos, bem como capacitações profissionais, tais como: educação ambiental e auditoria interna.
Segundo Corrêa et al. (2007), é preciso dar mais atenção para esta temática nos cursos de graduação da área de saúde, saindo do apenas saber como fazer e proporcionando reflexão, crítica, problematização, comprometimento com atuação baseada em ética, comprometimento social, solidariedade permitindo a este profissional uma atuação responsável com o meio. Já para Garcia e Zanetti-Ramos (2004), os profissionais devem não só realizar a segregação de forma correta e garantir a disposição final adequada, mas também nortear a população geral quando a segregação dos resíduos fora dos ambientes de saúde, promovendo saúde para toda comunidade.

Em relação à comparação significativa entre a capacitação, a Tabela 3 demonstrou que houve aumento significativo na melhora do descarte adequado dos resíduos, diminuindo a presença de resíduos infectantes nos coletores para resíduos comuns dos setores participantes da pesquisa, exceto no setor de Obstetrícia clínica.

Quando 3 - Comparação significativa pelo teste T de student de resíduos coletados nas lixeiras de RSS comuns em KG, antes e após a capacitação profissional realizada no Hospital São Luiz

\begin{tabular}{|c|c|c|c|c|c|c|}
\hline \multirow[b]{2}{*}{ Unidade Geradora } & \multirow[b]{2}{*}{ Resíduo } & \multicolumn{2}{|c|}{ Antes } & \multicolumn{2}{|c|}{ Após } & \multirow[b]{2}{*}{$\mathbf{T}$} \\
\hline & & $\sum$ & $\bar{X}$ & $\sum$ & $\bar{X}$ & \\
\hline \multirow{2}{*}{ Obstetrícia cirúrgica } & Comum & 85,5 & 10,68 & 93,5 & 11,68 & $1,52 \mathrm{~ns}$ \\
\hline & Infectante & 9,3 & 1,16 & 4,5 & 0,56 & $4,98^{*}$ \\
\hline \multirow{2}{*}{ Obstetrícia médica } & Comum & 92,85 & 11,6 & 84,1 & 10,51 & $2,11^{\mathrm{ns}}$ \\
\hline & Infectante & 10,5 & 1,31 & 9,3 & 1,16 & $1,46^{\mathrm{ns}}$ \\
\hline \multirow[b]{2}{*}{ Clínica médica } & Comum & 93,5 & 11,68 & 79,6 & 9,95 & $4,25^{*}$ \\
\hline & Infectante & 18,1 & 2,26 & 10,4 & 1,3 & $9,63^{*}$ \\
\hline \multirow{2}{*}{ UTI adulto } & Comum & 79,96 & 9,62 & 101 & 12,62 & $4,42^{*}$ \\
\hline & Infectante & 12,78 & 1,59 & 4,9 & 0,61 & $10,36^{*}$ \\
\hline \multirow{2}{*}{ UTI neonatal } & Comum & 52,8 & 6,6 & 51,2 & 6,4 & $1,95^{\mathrm{ns}}$ \\
\hline & Infectante & 9,3 & 1,16 & 5,1 & 0,63 & $3,43^{*}$ \\
\hline
\end{tabular}

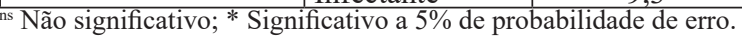

Fonte: Dados da pesquisa. 
De acordo com os dados estatísticos, o total de resíduos comuns descartados, nos resíduos infectantes, é maior do que o total de resíduos infectantes descartados no comum. $\mathrm{O}$ que não minimiza os agravos causados como consequência do manejo inadequado, pois pode ocasionar em impactos econômicos ao hospital e ambientais para a sociedade.

Pode-se perceber que a quantidade de geração dos resíduos sólidos, pelos setores, não se distanciou em relação aos que pertencem ao grupo A e aos que pertencem ao grupo D. No entanto, quando segregados de maneira correta durante a pesquisa, observou-se que esses resíduos são segregados de maneira ineficaz, gerando uma diferença na quantidade de resíduos.

Pode-se perceber que em relação aos resíduos orgânicos, estes são descartados em menores quantidades nos resíduos infectantes, quando comparados à quantidade descartada nos resíduos comuns. O descarte inadequado dos resíduos comuns juntamente com infecciosos foi observado em todos os setores analisados. Tal fato sugere que os profissionais do hospital estudado não recebiam treinamento adequado sobre destinação de resíduos.

O resultado dessa prática reduz os gastos com tratamento de resíduos, colaborando com os gastos da instituição, os impactos ao meio ambiente e para a saúde da população, principalmente, dos profissionais envolvido com o manuseio dos resíduos descartados, assim é necessário um programa de capacitação mais eficiente. Para Fernandes et al. (2007), o programa de capacitação como o PGRSS, ou educação com palestras educativas e entre outros permanentes, são importantes, pois é através destes que os profissionais do hospital podem gerar a familiarização com as normas, que regem todo o gerenciamento dos resíduos, tanto quanto aderir aos princípios que visam manutenção da saúde do trabalhador, da comunidade e a preservação do meio ambiente.

Segundo o Centro Nacional de Tecnologias Limpas (1998), a redução da geração de resíduos é possível com projetos de redução de gastos e educação ambiental voltados para a conscientização, com o desenvolvimento de tecnologias "limpas" de produção, redução de embalagens, combate ao desperdício e ampliação do reaproveitamento e reciclagem.

\section{Conclusão}

Mesmo que o estabelecimento forneça toda a estrutura adequada e disponibilize coletores para que a separação dos resíduos seja realizada, de maneira correta, ainda assim, não é suficiente para minimizar os prejuízos econômicos e ambientais, pois os profissionais devem estar trabalhando de acordo com os procedimentos estabelecidos no PGRSS, sendo assim é necessário um processo contínuo de capacitações profissionais aos funcionários e responsáveis pela geração e manuseio dos resíduos, bem como a vistoria periódica quanto ao manejo dos resíduos, assim poderá minimizar os riscos de acidentes de trabalho.

\section{Referências}

BRASIL. Lei $\mathrm{n}^{\mathrm{o}}$ 12.305, de 2 de agosto de 2010. Institui a Política Nacional de Resíduos Sólidos; altera a Lei no 9.605, de 12 de fevereiro de 1998; e dá outras providências. 2010.

BRASIL. Manual de gerenciamento de resíduos de serviços de saúde. Ministério da Saúde, Agência Nacional de Vigilância Sanitária - ANVISA. Brasília: Ministério da Saúde, 2006.

BRASIL. Resolução ANVISA RDC n ${ }^{\circ}$ 306, de 7 de dezembro de 2004. Dispõe sobre o Regulamento Técnico para o gerenciamento de resíduos de serviços de saúde. 2004

BRASIL. Resolução CONAMA No 05/1993. Define as normas mínimas para tratamento de resíduos sólidos oriundos de serviços de saúde, portos e aeroportos e terminais rodoviários e ferroviários. 1993.

CENTRO NACIONAL DE TECNOLOGIAS LIMPAS. Produção limpa: uma abordagem ambiental e econômica para a indústria. n.1. Pesquisa, p. 24. Ação Ambiental, Viçosa, 1998.

COELHO, H. Rejeitos em saúde: como descartar, aproveitar e gerenciar. Rio de Janeiro: Associação Nacional de Biossegurança, 2003.

COELHO, H. Manual de gerenciamento de resíduos de serviços de saúde. Rio de Janeiro: Fiocruz, 2000.

CORREA, L. B.; LUNARDI, V. L.; DE CONTO, S. M. O processo de formação em saúde: o saber resíduos sólidos de serviços de saúde em vivências práticas. Rev. Bras. Enferm., v.60, n.1, p.21-25, 2007.

DIAS, S.M.F.; FIGUEIREDO, L.C.A educação ambiental como estratégia para a redução da geração de resíduos de serviços de saúde em hospital de Feira de Santana. In: CONGRESSO BRASILEIRO DE ENGENHARIA SANITÁRIA E AMBIENTAL, 20, 1999, Rio de Janeiro. Anais... p. 3608-17. Rio de Janeiro: ABES, 1999.

FERNANDES, M.I.; MACIEL, S.S.S.V.; XAVIER, W.C.S Gerenciamento dos resíduos sólidos nos serviços de saúde dos hospitais de Caruaru-PE. Rev. Saúde Coletiva, v.3, n.1, p.45-54, 2007.

GARCIA, L.P.; ZANETTI-RAMOS, B.G. Gerenciamento dos resíduos de serviços de saúde: uma questão de biossegurança Cad. Saúde Pública, v.20, n.3, p.744-752, 2004.

NAIME, R.; RAMALHO, A.H.P.; NAIME, I.S. Avaliação do Sistema de Gestão dos Resíduos Sólidos do Hospital de Clínicas de Porto Alegre. Rev. Espaço Saúde, v.9, n.1, p.1-17, 2008.

SCHNEIDER, V.E. et al. Modelos de gestão de resíduos sólidos de serviços odontológicos (RSSO) por meio do índice de geração per capita. In: CONGRESO INTERAMERICANO DE INGENIERÍA SANITARIA Y AMBIENTAL, Cancun (México). Anais... v.28, 2002.

SILVA, C.E.; HOPPE, A.E. Diagnóstico dos resíduos de serviços de saúde no interior do Rio Grande do Sul. Eng. Sanitária Amb., v.10, n.2, p.146-151, 2005.

SOUZA, F.P. et al. Viabilidade da Aplicação da Logística Reversa no Gerenciamento dos Resíduos dos Serviços de Saúde: um Estudo de Caso no Hospital X. Perspectivas Online: Ciênc. Exatas Eng., v.3, n.6, p.56-72, 2006. 